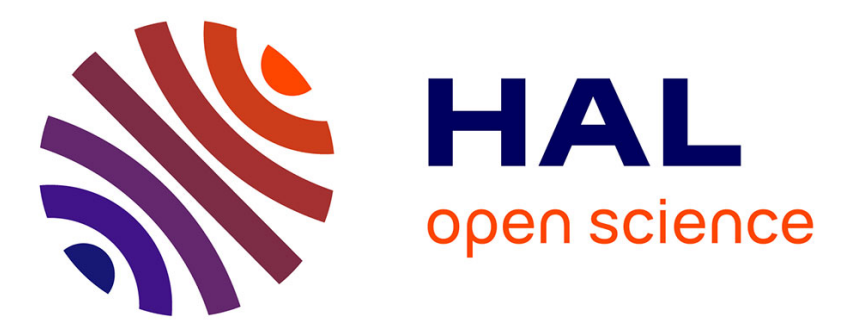

\title{
Low-Complexity Half-Sparse Decomposition-based Detection for massive MIMO Transmission
}

Zahran Hajji, Karine Amis Cavalec, Abdeldjalil Aissa El Bey, Fatma Abdelkefi

\section{To cite this version:}

Zahran Hajji, Karine Amis Cavalec, Abdeldjalil Aissa El Bey, Fatma Abdelkefi. Low-Complexity HalfSparse Decomposition-based Detection for massive MIMO Transmission. 5th International Conference on Communications and Networking (ComNet 2015), Nov 2015, Hammamet, Tunisia. pp.1-6. hal01308364

\section{HAL Id: hal-01308364 https://hal.science/hal-01308364}

Submitted on 27 Apr 2016

HAL is a multi-disciplinary open access archive for the deposit and dissemination of scientific research documents, whether they are published or not. The documents may come from teaching and research institutions in France or abroad, or from public or private research centers.
L'archive ouverte pluridisciplinaire HAL, est destinée au dépôt et à la diffusion de documents scientifiques de niveau recherche, publiés ou non, émanant des établissements d'enseignement et de recherche français ou étrangers, des laboratoires publics ou privés. 


\title{
Low-Complexity Half-Sparse Decomposition-based Detection for massive MIMO Transmission
}

\author{
Zahran Hajji*†, Karine Amis*, Abdeldjalil Aïssa-El-Bey* and Fatma Abdelkefi ${ }^{\dagger}$ \\ *Institut Mines-Telecom, Telecom Bretagne, Lab-STICC, UMR CNRS 6285, 29238 Brest, France \\ Université européenne de Bretagne \\ Email: Firstname.Lastname@telecom-bretagne.eu \\ ${ }^{\dagger}$ Mediatron laboratory, Sup'Com, Tunisia \\ Email: Firstname.Lastname@supcom.tn
}

\begin{abstract}
This paper focuses on low-complexity detection for large scale multiple-input multiple-output (MIMO) systems involving tens to hundreds of transmit/receive antennas. Due to the exponential increase of its processing complexity with the data signal dimensions (antenna number, modulation order), a maximum likelihood detection is infeasible in practice. To overcome this drawback, authors in [1] proposed a lowcomplexity detection based on a sparse decomposition of the information vector. It is proved that this decomposition is mainly adpated to underdetermined systems and leads to a significant reduction on the processing complexity. As an extension to the work investigated in [1], we propose in this paper a new decomposition that makes the computation cost less dependent on the modulation alphabet cardinality, thus reducing theoretically the complexity by $50 \%$ for $4-\mathbf{Q A M}$ and by $72 \%$ for $16-\mathbf{Q A M}$ compared to the previous detector in [1], while achieving the same error rate performance.
\end{abstract}

Index Terms-Sparse representation, source separation, basis pursuit, maximum likelihood detection, massive MIMO...

\section{INTRODUCTION}

$\mathrm{D}$ ATA traffic is expected to significantly increase in the next decade due to the increase of the connected machine number (machine type communications) and the data rate. MIMO technology has been selected in the $5 \mathrm{G}$ standard definition as a solution to provide higher throughput under spectrum limitations [2]. Large number of antennas and/or users is involved, which makes the receiver design critical from the complexity point of view. The maximum likelihood (ML) joint detection is optimum in term of bit error rate (BER) but its complexity increases exponentially with the system dimensions and the modulation alphabet cardinality which makes it infeasible in practice [3]. The sphere decoder reduces the search space to a hypersphere around the signal-space projection of the received signal and performs near-optimally provided the hypersphere radius be well tuned [4]. However, sphere decoding technique involves an exhaustive search within the hypersphere whose dimensions remain high in the large-scale MIMO case, yielding computationally-unsolvable detection. Research for high-performance receiver design that can lead to practical realization of large- MIM0 systems is both nascent as well as promising [5]. Usual linear equalizers such as minimum mean square error (MMSE) and zero-forcing (ZF) have low computation complexity but perform poorly when used in underdetermined uncoded MIMO systems. Such configuration is expected in future $5 \mathrm{G}$ system uplink, as the number of connected users times their transmit antenna number could be much higher than the base station receive antenna number. Previous works proved that source separation is possible in the underdetermined case thanks to basis pursuit (BP) technique [6]. Following this approach, a sparse representation was proposed in [7] to define a successful separation method for a conditioned dimension system. Underdetermined noisy MIMO system with finite alphabet was dealt with as an application case of [7] in [1] and [8], where the problem was formulated as a basis pursuit denoising (BPDN) problem with relaxed constraints. In [8], the $\ell_{0}$-norm was relaxed into the $\ell_{1}$ norm to obtain a minimization problem which is solved thanks to an iterative algorithm subject to a spherical search space. The problem dependency on the sphere radius makes it harder to solve. The problem formulation proposed in [1] outperforms the previous one and its success detection probability relies only on the equivalence between the $\ell_{0}$-norm and $\ell_{1}$-norm. This formulation is based on a ML criterion applied with relaxed constraints. In this paper, we consider the method developed in [1] and we look for a new basis with limited size in order to develop a more reduced complexity algorithm and maintain a low computational cost even with the increase of the system and/or constellation size. This proposed algorithm relies on a half-sparse decomposition of the data signal vector and a new problem formulation.

This paper is organized as follows. Section II describes the underdetermined MIMO transmission model. Section III deals with the source separation problem in the noiseless case. We propose the new half-sparse decomposition and we introduce the corresponding problem formulation. In Section IV, we apply the proposed scheme to the massive MIMO noisy channel. In Section $\mathrm{V}$, we compare the proposed half-sparse decomposition-based detection method to the one investigated in [1] as well as to the MMSE linear equalizer. Finally, Section VI concludes the paper.

Notations: boldface upper case letters and boldface lower case letters denote matrices and vectors, respectively. For the transpose, transpose conjugate and conjugate matrices we use $(.)^{T},(.)^{H}$ and $(.)^{*}$, respectively. $\otimes$ is the Kronecker product. $\boldsymbol{I}_{p}$ is the $p \times p$ identity matrix and $\mathbf{1}_{p}$ is the all-one size- $p$ vector. 


\section{SYSTEM MODEL}

Let us consider a $K$-user MIMO transmission over a flat fading channel, where each user has $N_{t}$ transmit antennas and $N_{r}$ receive antennas and let $N=K \times N_{t}$. No user cooperation for transmission nor precoding scheme is taken into account. At the base station side, we assume $n$ equivalent receive antennas (base station cooperation through the base station controller is possible) with $n \leq N$. We assume a perfect knowledge of the channel state information (CSI) at the base station. In this paper, we focus on the uplink of the communication system. The received signal is defined as follows:

$$
\boldsymbol{y}=\boldsymbol{H} \boldsymbol{x}+\boldsymbol{z} .
$$

where $\boldsymbol{H}$ is an $n \times N$ random channel matrix, $x$ is the $N \times 1$ data vector, and $z$ is the $n \times 1$ complex circularly symmetric additive Gaussian noise vector with zero mean and a covariance matrix equal to $\sigma^{2} \boldsymbol{I}$. We assume that the components of $x$ belong to a $M$-QAM modulation alphabet such that $M=L^{2}$ (square QAM constellation). In that case denoting by $\mathcal{Q}$ the modulation alphabet then $\mathcal{Q}=\left\{q_{1}, q_{2}, \ldots, q_{M}\right\}$. We associate to $Q$ the symbol vector $\boldsymbol{q}=\left[q_{1}, q_{2}, \ldots, q_{M}\right]$ where $q_{i}=a_{i}+j b_{i}, i \in\{1 . . M\}$ with $\left(a_{i}, b_{i}\right) \in \mathcal{A} \times \mathcal{A}$ and $\mathcal{A}=\left\{-2^{k}+1, \ldots,-3,-1,1,3, \ldots, 2^{k}-1\right\}, k=\log _{2}(\sqrt{M})$.

The challenge is to design an efficient joint detection scheme of the transmitted data with moderate computational complexity order over the whole SNR range in an underdetermined system $(n \leq N)$. In the following, we propose to recover $x$ from sparse detection techniques as described in [1] but with a new decomposition that enables a computation cost reduction while keeping the same error rate performance. To this end, in Section III we study the detection scheme with the new decomposition assuming a noise-free transmission. Then, in section IV, we extend it to the case of the noisy massive MIMO transmission uplink described by (1).

\section{SPARSITY-BASED RECOVERY METHOD (NOISE-FREE)}

\section{A. Problem statement}

We consider the underdetermined linear system or noise free mixing model

$$
\boldsymbol{y}=\boldsymbol{H} \boldsymbol{x}, \boldsymbol{x} \in \mathrm{Q}^{N} .
$$

where $\boldsymbol{x}=\left[x_{1}, x_{2}, \ldots, x_{N}\right]^{T}$ is the $N \times 1$ source vector, $\boldsymbol{y}=\left[y_{1}, y_{2}, \ldots, y_{n}\right]^{T}$ is the $n \times 1$ observation vector and $\boldsymbol{H}$ is an $n \times N$ generic random matrix with $n \leq N$. In [1], [8], the authors proposed a framework for the recovery of finite alphabet signals based on their sparse decomposition.

\section{B. Existing sparse decomposition [1]}

Exploiting the fact that the vector $\boldsymbol{x}$ belongs to a finite alphabet, each element of $\boldsymbol{x}$ can be decomposed on the basis of the vector space in which the finite alphabet vector $\boldsymbol{q}=\left[q_{1}, q_{2}, \ldots, q_{M}\right]$ can be cast. The data vector with $N$ entries can be modeled as an equivalent sparse data vector with $N M$ entries.

The decomposition of the $j^{t h}$ component of $\boldsymbol{x}$ is decomposed as

$$
\begin{aligned}
x_{j} & =\boldsymbol{q} \boldsymbol{r}_{j}^{T}, \\
\text { where } \boldsymbol{r}_{j} & =\left[\delta_{q_{1}}\left(x_{j}\right), \delta_{q_{2}}\left(x_{j}\right), \ldots, \delta_{q_{M}}\left(x_{j}\right)\right] .
\end{aligned}
$$

Applying this decomposition over all the components, the vector $\boldsymbol{x}$ can be formulated as a function of $\boldsymbol{r}$ as

$$
\begin{aligned}
\boldsymbol{x} & =\boldsymbol{B}_{q} \boldsymbol{r}, \\
\text { where } \boldsymbol{r} & =\left[r_{1}, r_{2}, \ldots, r_{N}\right]^{T}, \\
\boldsymbol{B}_{q} & =\boldsymbol{I}_{N} \otimes \boldsymbol{q} .
\end{aligned}
$$

$\boldsymbol{B}_{q}$ is a $N \times N M$ block diagonal matrix.

Then, the received signal is written as

$$
\boldsymbol{y}=\boldsymbol{H} \boldsymbol{B}_{q} \boldsymbol{r}
$$

\section{Proposed half-sparse decomposition}

In this section we propose another sparse decomposition which reduces the complexity of the decoding scheme as compared to the first decomposition. We consider $M$-QAM modulations and the sets $\mathcal{Q}$ and $\mathcal{A}$ defined in Section II. We propose to decompose the elements of $\mathcal{A}$ on a basis with binary components vector as introduced in [9]. Let $\boldsymbol{\beta}=\left[2^{k-1}, \ldots, 2^{1}, 2^{0}\right]$. Then, each component of $\mathcal{A}$ can be decomposed as

$$
a=\boldsymbol{\beta} \boldsymbol{c}^{T} \text {, where } \boldsymbol{c} \in\{-1,1\}^{k} .
$$

As $c \in\{-1,1\}^{k}$, its $i^{\text {th }}$ component, denoted by $c_{i}$, can be written as

$$
\begin{aligned}
c_{i} & =\boldsymbol{p} \boldsymbol{s}_{i}^{T}, \\
\text { where } \boldsymbol{p} & =[-1,1], \\
\text { and } \quad \boldsymbol{s}_{i} & =\left[\delta_{(-1)}\left(c_{i}\right), \delta_{(1)}\left(c_{i}\right)\right] .
\end{aligned}
$$

Let $\boldsymbol{\beta}_{c}=[\boldsymbol{\beta} j \boldsymbol{\beta}]$. Extending the previous decomposition to Q, each element $q$ of $\mathcal{Q}$ can be expressed as:

$q=a+j b=\boldsymbol{\beta}_{c} \boldsymbol{c}^{T}$, with $\boldsymbol{c} \in\{-1,1\}^{\ell}$, and $\quad \ell=\log _{2}(M)=2 k$.

As $c \in\{-1,1\}^{\ell}$, the $i^{t h}$ element of $c$ can be written as follows

$$
\begin{aligned}
c_{i} & =\boldsymbol{p} \boldsymbol{s}_{i}^{T}, \\
\text { where } \quad \boldsymbol{p} & =[-1,1] \\
\text { and } \quad \boldsymbol{s}_{i} & =\left[\delta_{(-1)}\left(c_{i}\right), \delta_{(1)}\left(c_{i}\right)\right] .
\end{aligned}
$$

Coming back to the system model, the vector $\boldsymbol{x}$ can be formulated as a function of $s$

$$
\begin{aligned}
\boldsymbol{x} & =\boldsymbol{B}_{\beta} \boldsymbol{B}_{p} \boldsymbol{s}, \\
\text { with } \quad \boldsymbol{B}_{\beta} & =\boldsymbol{I}_{N} \otimes \boldsymbol{\beta}_{c}^{T}, \\
\boldsymbol{B}_{p} & =\boldsymbol{I}_{\ell N} \otimes \boldsymbol{p}, \\
\boldsymbol{s} & =\left[\boldsymbol{s}_{1}, \boldsymbol{s}_{2}, \ldots, \boldsymbol{s}_{\ell N}\right]^{T} \in\{0,1\}^{2 \ell N} .
\end{aligned}
$$

where $\boldsymbol{B}_{\beta}$ is a $N \times \ell N$ block diagonal matrix and $\boldsymbol{B}_{p}$ is a $\ell N \times 2 \ell N$ block diagonal matrix.

It results that the received signal reads

$$
\boldsymbol{y}=\boldsymbol{H} \boldsymbol{B}_{\beta} \boldsymbol{B}_{p} \boldsymbol{s}
$$




\section{Source separation}

Since the output of the first decomposition is a sparse vector that contains a majority of zero elements, the detection of the original information can be seen as a sparse source decoding. In this context, the authors in [7] demonstrated that the vector $x$ can be recovered by resolving the following optimization problem:

$$
\left(P_{\mathcal{F}, 1}\right): \underset{r}{\arg \min }\|\boldsymbol{r}\|_{1} \text { subject to } \boldsymbol{r} \in \mathcal{F},
$$

where $\mathcal{F}=\left\{\boldsymbol{r} \mid \boldsymbol{y}=\boldsymbol{H} \boldsymbol{B}_{q} \boldsymbol{r}, \boldsymbol{B}_{1}^{\mathcal{F}} \boldsymbol{r}=\mathbf{1}_{N}\right\}$ and $\boldsymbol{B}_{1}^{\mathcal{F}}=\boldsymbol{I}_{N} \otimes \mathbf{1}_{M}$.

Considering the new decomposition (10), the vector $s$ is half sparse as half of its components are equal to zero. We can still apply sparse detection techniques to recover the original information. The new optimization problem reads:

$$
\left(P_{\mathcal{G}, 1}\right): \underset{s}{\arg \min }\|\boldsymbol{s}\|_{1} \text { subject to } \boldsymbol{s} \in \mathcal{G},
$$

where $\mathcal{G}=\left\{\boldsymbol{s} \mid \boldsymbol{y}=\boldsymbol{H} \boldsymbol{B}_{\beta} \boldsymbol{B}_{p} s, \boldsymbol{B}_{1}^{\mathcal{G}} \boldsymbol{s}=\mathbf{1}_{\ell N}\right\}$ and $\boldsymbol{B}_{1}^{\mathcal{G}}=\boldsymbol{I}_{\ell N} \otimes \mathbf{1}_{2}$.

\section{EXTENSION TO THE NOISY MASSIVE MIMO TRANSMISSION}

We now consider the MIMO system described in Section II with large values of $n$ and $N$.i.e. (massive MIMO configuration). In this respect, the optimal ML joint detection, based on exhaustive search is a problem computationally unsolvable. Using the first decomposition, the received signal can be defined as follows [1]:

$$
\boldsymbol{y}=\boldsymbol{H} \boldsymbol{B}_{q} \boldsymbol{r}+\boldsymbol{z} .
$$

To recover the sparse vector $\boldsymbol{r}$, the authors in [7] proposed to solve the following optimization problem:

$$
\begin{aligned}
\left.\underset{P_{\mathcal{F}, 2}}{ }\right): \underset{r}{\arg \min } & \left\|\boldsymbol{y}-\boldsymbol{H} \boldsymbol{B}_{q} r\right\|_{2} \\
\text { subject to } \boldsymbol{B}_{1}^{\mathcal{F}} \boldsymbol{r} & =\mathbf{1}_{N}, \\
\boldsymbol{r} & \geq 0 .
\end{aligned}
$$

This optimization problem statement can be seen as a ML relaxed detector that minimizes the euclidean distance with the received signal. When we apply the proposed half-sparse decomposition, the received signal can be written as:

$$
\boldsymbol{y}=\boldsymbol{H} \boldsymbol{B}_{\beta} \boldsymbol{B}_{p} \boldsymbol{s}+\boldsymbol{z} .
$$

Exploiting the half-sparsity of $s$, we can investigate the same approach as in [1]. Given (16), we seek the half-sparse vector $s$ that minimizes the euclidean distance to the received signal on a well-defined plane. The ML detector requires an exhaustive search through all the possible values of $s$. The solution corresponds to the closest point to $\boldsymbol{y}$ in the received constellation. Hence the ML detection problem reads

$$
\left(P_{M L}\right): \underset{x}{\arg \min } \quad\|\boldsymbol{y}-\boldsymbol{H} \boldsymbol{x}\|_{2} \quad \text { subject to } \boldsymbol{x} \in \mathbb{Q}^{N} .
$$

The main drawback of the ML detection problem remains its high computational complexity due to the constraint $\boldsymbol{x} \in Q^{N}$ that entails an exhaustive search.

In [1], the authors relaxed the ML problem into the minimization one $\left(P_{\mathcal{F}, 2}\right)$ that can be solved by using iterative algorithms with polynomial complexity. Following the same approach, we propose an equivalence to the constraint $\boldsymbol{x} \in Q^{N}$ using the following Proposition 1 . which resorts to the half-sparse decomposition defined in (10).

Proposition 1. The components of $\boldsymbol{x}$ belong to the finite alphabet constellation $Q$ if and only if the following equalities hold: $\boldsymbol{B}_{1}^{\mathcal{G}} s=\mathbf{1}_{\ell N}$ and $\|s\|_{0}=\ell N$

Proof. Let us assume that the vector $\boldsymbol{x}$ belongs to the finite alphabet constellation $\mathcal{Q}$, then it can be half-sparsely decomposed as $\boldsymbol{x}=\boldsymbol{B}_{\beta} \boldsymbol{B}_{p} s$, where $s$ is composed of $\ell N$ consecutive 2 -uples, such that each 2 -uple contains one 1 and one 0 . This means that $\boldsymbol{B}_{1} s=\mathbf{1}_{\ell N} . s$ is a half-sparse vector satisfying $\|s\|_{0}=\ell N$.

Reciprocally, let us now assume that $\boldsymbol{B}_{1}^{\mathcal{G}} \boldsymbol{s}=\mathbf{1}_{\ell N}$ and $\|s\|_{0}=\ell N . B_{1}^{\mathcal{S}} \boldsymbol{s}=\mathbf{1}_{\ell N}$ means that $s_{j}+s_{(j+1)}=1$ for all $j \in\{1,3, \ldots, 2 \ell N-1\}$, implying that at least one component of each 2-uple is different from zero. The second equality $\|s\|_{0}=\ell N$ imposes that the total number of non-zero elements in $s$ is equal to $\ell N$. Using the two previously deducted properties, we conclude that each 2-uple has exactly one non-zero component equal to 1 . Thereby, the length- $N$ vector $\boldsymbol{x}=\boldsymbol{B}_{\beta} \boldsymbol{B}_{p} s$ belongs to the finite alphabet $Q^{N}$.

Using Proposition 1., the minimization problem $\left(P_{M L}\right)$ becomes

$$
\begin{aligned}
\underset{s}{\arg \min } & \left\|\boldsymbol{y}-\boldsymbol{H} \boldsymbol{B}_{\beta} \boldsymbol{B}_{p} \boldsymbol{s}\right\|_{2} \\
\text { subject to } \boldsymbol{B}_{1}^{\mathcal{S}} \boldsymbol{s} & =\mathbf{1}_{\ell N}, \\
\|\boldsymbol{s}\|_{0} & =\ell N .
\end{aligned}
$$

The constraint $\|s\|_{0}=\ell N$ makes the problem NP-hard. It requires an exhaustive search to seek the solution. By exploiting the sparsity of the searched vector, we propose to relax the $\ell_{0}$-norm into the $\ell_{1}$-norm. The relaxed constraint becomes $\|s\|_{1}=\ell N$. However, our system is not convex, thus, a global optimum is not necessarily achieved. To overcome this problem, we introduce the following lemma:

Lemma 1. Let $\boldsymbol{B}_{1}^{\mathcal{G}}=\boldsymbol{I}_{\ell N} \otimes \mathbf{1}_{2}$ and $\boldsymbol{s}$ a $(2 \ell N) \times 1$ real vector satisfying $\boldsymbol{B}_{1}^{\mathcal{G}} s=\mathbf{1}_{\ell N}$. Then all components of $s$ are positive if and only if its $\ell_{1}$-norm equals $\ell N$ i.e. $\|s\|_{1}=\ell N$.

Proof. The equality $\boldsymbol{B}_{1}^{\mathcal{G}} \boldsymbol{s}=\mathbf{1}_{\ell N}$ implies that $s_{j}+s_{(j+1)}=1$ for all $j \in\{1,3, \ldots, 2 \ell N-1\}$.

By successive additions of the 2-uples which compose the sparse vector, we obtain

$$
\sum_{i=1}^{2 \ell N} s_{i}=\sum_{1 \leq j \leq \ell N, j \text { odd }} s_{j}+s_{(j+1)}=\ell N .
$$

Let us assume that all the components of $s$ are positive. We deduce that $\sum_{i=1}^{2 \ell N}\left|s_{i}\right|=\ell N$, i.e $\|s\|_{1}=\ell N$.

Reciprocally, let assume that $\|s\|_{1}=\ell N$. According to (19), we can thus write

$$
\sum_{i=1}^{2 \ell N}\left(\left|s_{i}\right|-s_{i}\right)=0
$$

Let $N(s)$ denote the set of indexes corresponding to all 
non-zero negative elements of $s$. Then $\sum_{i=1}^{2 \ell N}\left(\left|s_{i}\right|-s_{i}\right)=$ $2 \sum_{i \in N(s)}\left|s_{i}\right|$. It follows from (20) that $s_{i}=0$ when $i \in N(s)$ which is in contradiction with the definition of $N(s)$. We conclude that $N(s)=\emptyset$ and that all the components of $s$ are positive.

Using Lemma 1., the decoding problem becomes

$$
\begin{aligned}
\left(P_{\mathcal{G}, 2}\right): \underset{s}{\arg \min } & \left\|\boldsymbol{y}-\boldsymbol{H} \boldsymbol{B}_{\beta} \boldsymbol{B}_{p} \boldsymbol{s}\right\|_{2} \\
\text { subject to } \boldsymbol{B}_{1}^{\mathcal{G}} \boldsymbol{s} & =\mathbf{1}_{\ell N} \\
\boldsymbol{s} & \geq 0 .
\end{aligned}
$$

The new optimization problem $\left(P_{\mathcal{G}, 2}\right)$ is a quadratic programming model with linear equality constraints and nonnegative variables.

\section{Simulation Results}

In this section, we evaluate the new half-sparse decomposition-based detection in terms of complexity and error rate in the discussed cases in Section III (noiseless channel) and in Section IV (noisy channel). The channel coefficients are assumed to be i.i.d circularly symmetric complex Gaussian distributed with zero mean and unit variance. We use the CVX toolox, which is a Matlab library for convex optimization [10]. The $\ell_{1}$-minimization problem under convex constraints $\left(P_{\mathcal{G}, 1}\right)$ is solved by the SeDumi solver [11], while the quadratic minimization problem $\left(P_{\mathcal{G}, 2}\right)$ is solved by using the Gurobi optimizer [12]. The simulation results are obtained by using a PC with OS Linux Ubuntu 14.04 with processor Intel Core i3-2350M 2.3 GHz and $8 \mathrm{~GB}$ of RAM memory. For the sake of brievity, in the remainder of the paper, we refer to each detector by the problem that solves. For example, the detector that seeks for the solution of $\left(P_{\mathcal{G}, 1}\right)$ will be referred to as $\left(P_{\mathcal{G}, 1}\right)$ detector.

\section{A. Noiseless MIMO channel}

In order to study the performance of the proposed detection over the noiseless channel, we compare the solution of the optimization problem $\left(P_{\mathcal{G}, 1}\right)$ found by the SeDumi solver, denoted by $\hat{\boldsymbol{x}}$, with the true signal $\boldsymbol{x}$. The recovery is said to be correct if the relative error $\frac{\|\hat{\boldsymbol{x}}-\boldsymbol{x}\|_{2}}{\|\boldsymbol{x}\|_{2}}$ is less than $10^{-5}$ [7]. The phase diagrams are plotted in Fig. 1 for $M=4,16$ and 64. We observe that the half-sparse decomposition enables to achieve the same performance as the original sparse decomposition. Moreover, it can be observed that the success probabilility is higher than $50 \%$ when $\frac{\sqrt{M}-1}{\sqrt{M}} n \leq N$ and tends to 1 when $\frac{n}{N}$ increases with a higher convergence rate for large values of $N$. We deduce the asymptotic equivalence between the $\ell_{0}$ and $\ell_{1}$-based optimization problems. According to Fig. 1 we observe that the success probability increases from $50 \%$ to $100 \%$ with a slightly variation of $\frac{n}{N}$ for higher $N$. For the 4-QAM the optimum convergence state is obtained for a variation $\Delta \frac{n}{N}=0.055$ when $N=256$ and it is equal to 0.09 for $N=128$.

The interest of the new half-sparse decomposition can be motivated by its complexity order. The CVX toolbox relies

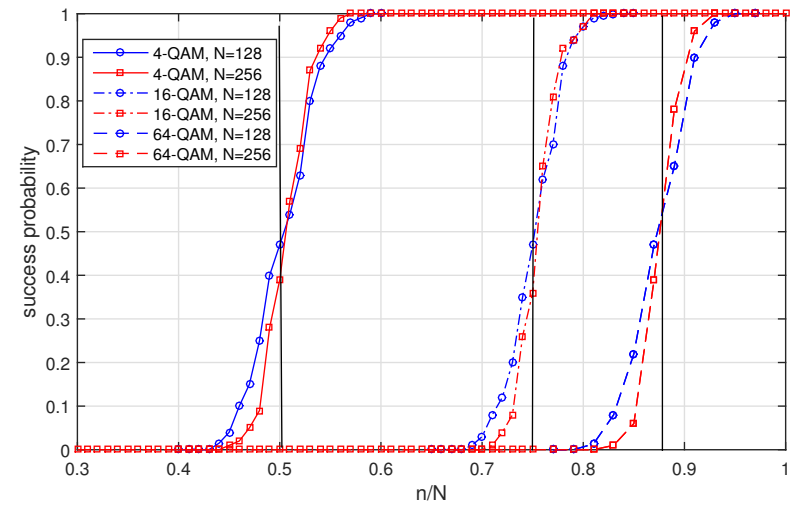

Fig. 1: Phase diagrams of the half-sparse decomposition-based detection for $M$-QAM with $M=4,16,64$ and $N=128,256$. (Solution of $\left(P_{\mathcal{G}, 1}\right)$ found by the CVX SeDumi solver)

on the interior point method whose complexity is a function of the number of constraints and the dimension of the searched vector. A convex optimization problem defined in the real field over $\mathbb{R}^{m}$ under $d$ constraints requires, in the worst case, $O(\sqrt{d})$ iterations for a computational cost of order $O\left(m^{2} d\right)$ per iteration and yields a total computational cost of order $O\left(m^{2} d^{3 / 2}\right)$ [13]. Applied to $\left(P_{\mathcal{F}, 1}\right)$ and $\left(P_{\mathcal{G}, 1}\right)$, we obtain the computational costs given in Table 1 . The total computation cost is $\left(\frac{M}{\log _{2}(M)}\right)^{2}$ higher in the case of $\left(P_{\mathcal{F}, 1}\right)$, which makes the problem $\left(P_{\mathcal{G}, 1}\right)$ all the more interesting one to solve when the modulation order is high.

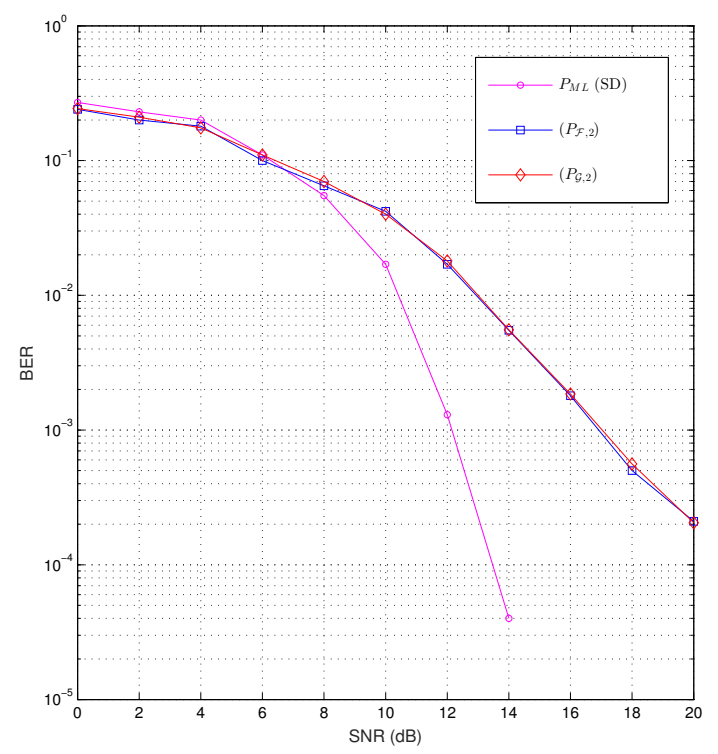

Fig. 2: BER performance comparison in $16 \times 14 \mathrm{MIMO}$ channel.

\section{B. Noisy MIMO channel}

For noisy massive MIMO transmission, Fig. 2 compares the BER performance of the proposed $\left(P_{\mathcal{G}, 2}\right)$ detection to the 


\begin{tabular}{|l|c|c|l|}
\hline & Dimension & Computational cost per iteration & Total \\
\hline$\left(P_{\mathcal{F}, 1}\right)$ & $M N$ & $O\left(M^{2} N^{2}(N+n)\right)$ & $O\left(M^{2} N^{2}(N+n)^{3 / 2}\right)$ \\
\hline$\left(P_{\mathcal{G}, 1}\right)$ & $\log _{2}(M) N$ & $O\left(\left(\log _{2}(M)\right)^{2} N^{2}(N+n)\right)$ & $O\left(\left(\log _{2}(M)\right)^{2} N^{2}(N+n)^{3 / 2}\right)$ \\
\hline
\end{tabular}

TABLE I: Computational cost with the interior point method.

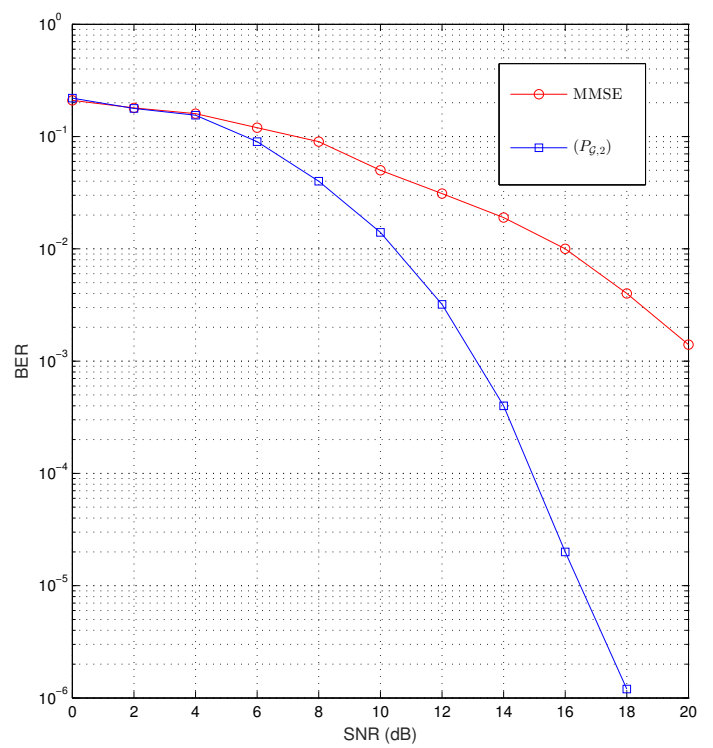

Fig. 3: BER performance comparison in $64 \times 64 \mathrm{MIMO}$ channel.

sphere decoder $(\mathrm{SD})$, described in [14], and to the $\left(P_{\mathcal{F}, 2}\right)$ detector. We assume a 4-QAM constellation mapping. As $\left(P_{\mathcal{F}, 2}\right)$ and $\left(P_{\mathcal{G}, 2}\right)$ resort to the same detection principle, they perform equivalently for different system dimensions. It can be also observed that beyond $6.7 \mathrm{~dB}$, the SD outperforms the $\left(P_{\mathcal{G}, 2}\right)$ detector, e.g. at BER $10^{-3}$, a gain of about $5 \mathrm{~dB}$ is achieved in the case of $16 \times 14 \mathrm{MIMO}$ system. Increasing the system dimension to $64 \times 64$, the SD complexity becomes too high and we compare in Fig. 3 the $\left(P_{\mathcal{G}, 2}\right)$ detector to the minimum mean square error (MMSE) detector. We remind that the MMSE detector requires that the system is at least determined. One can observe that the $\left(P_{\mathcal{G}, 2}\right)$ detector exploits better the receive diversity than the MMSE detector. At BER $10^{-2}$, the $\left(P_{\mathcal{G}, 2}\right)$ outperforms the MMSE by about $5.5 \mathrm{~dB}$. This gain increases to reach $7 \mathrm{~dB}$ for a BER of $10^{-2}$.

It is important to emphasize that in practice, an outer forward error correction (FEC) code is used and the proposed detection can be inserted within an iterative turbo-like receiver. In that case, the performance loss towards the SD can be reduced at the convergence state of the receiver.

Fig. 4 and Fig. 5 illustrate the runtime of the $\left(P_{\mathcal{G}, 2}\right)$ detection compared to the $\left(P_{\mathcal{F}, 2}\right)$ detection for 16-QAM and 64QAM respectively to show the asset of the new decomposition. It can be observed that the runtime increases slightly with the system dimension and independently of the SNR level. Moreover, we observe that the $\left(P_{\mathcal{G}, 2}\right)$ is computationally less costly than the $\left(P_{\mathcal{F}, 2}\right)$ e.g. for 16-QAM we reduce around $50 \%$ of the time run for any considered dimension value.

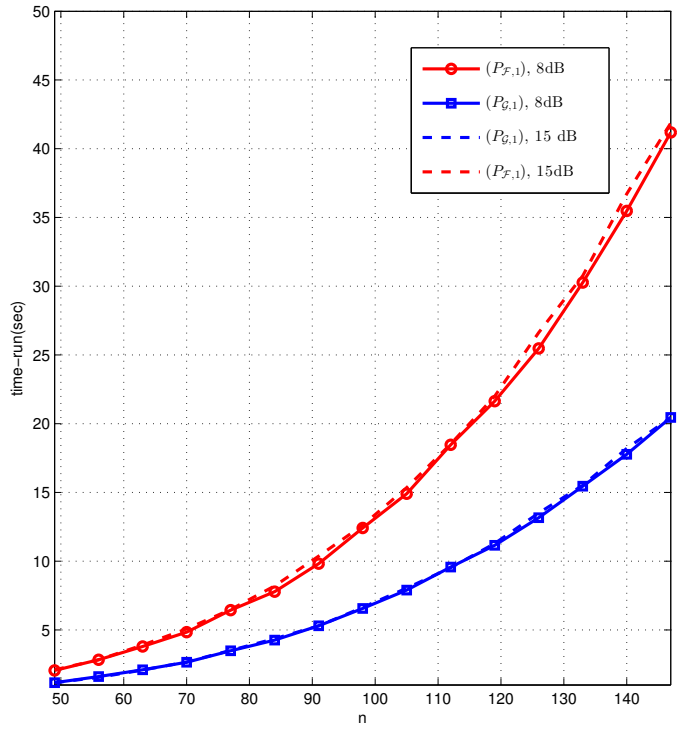

Fig. 4: Runtime comparison for different SNR values with a 16-QAM modulation and $\mathrm{N}=(8 / 7) \mathrm{n}$.

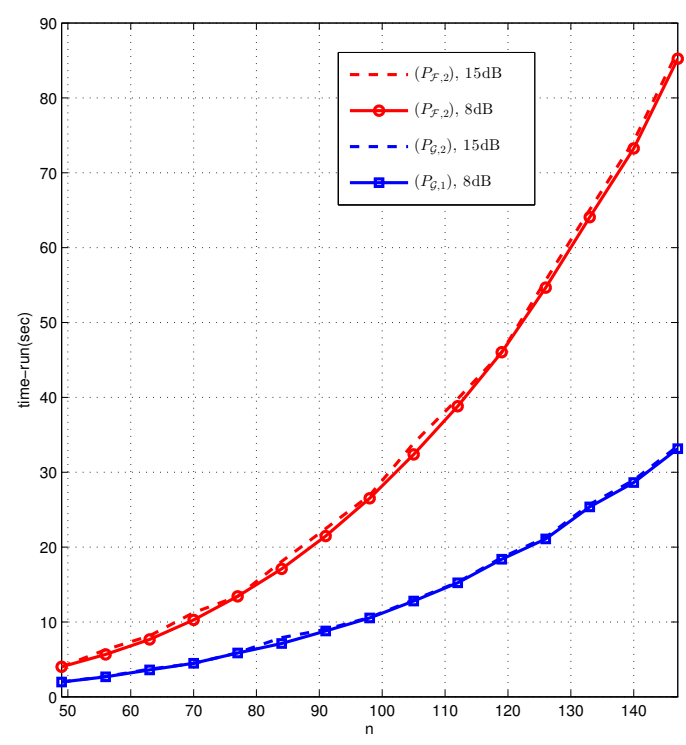

Fig. 5: Runtime comparison for different SNR values with a 64-QAM modulation and $\mathrm{N}=(8 / 7) \mathrm{n}$.

We also note from Fig. 4 and Fig. 5 that the gain in the computational cost compared to $\left(P_{\mathcal{F}, 2}\right)$ increases with the modulation order which is in agreement with the Table 1. This gain is around $72 \%$ for 64-QAM modulation. 
Let us emphasize that compared to the sparse decomposition used in $\left(P_{\mathcal{F}, 2}\right)$, the new half-sparse decomposition used in $\left(P_{\mathcal{G}, 2}\right)$ enables the detector to provide soft output that can be more directly exploited in a symbol to binary conversion to feed the FEC decoder with reliable soft input. The study is still in progress and preliminary results are promising.

\section{CONCLUSION}

In this paper we have addressed the problem of decoding in large MIMO systems with finite $M$-ary QAM constellation. We have proposed a new decomposition of the data symbol vector into a half-sparse input vector with half of its components equal to 0 . Exploiting its sparsity, we have relaxed the ML problem into another minimization problem $\left(P_{\mathcal{G}, 2}\right)$ of lower polynomial complexity compared to the original $\left(P_{\mathcal{F}, 2}\right)$, while achieving the same error rate performance. In addition to its asset for the complexity, a study still in progress shows that the new half-sparse decomposition enables the detector to provide reliable soft-output which can be integrated in a turbo-detection scheme.

\section{REFERENCES}

[1] Y. Fadlallah, A. Aissa-El-Bey, K. Amis, D. Pastor, and R. Pyndiah, "New iterative detector of mimo transmission using sparse decomposition," IEEE Transactions on Vehicular Technology, vol. PP, no. 99, pp. 1-1, 2014.

[2] B. Panzner, W. Zirwas, S. Dierks, M. Lauridsen, P. Mogensen, K. Pajukoski, and D. Miao, "Deployment and implementation strategies for massive mimo in 5g," in 2014 Globecom Workshops (GC Wkshps), Dec 2014, pp. 346-351.

[3] M. Damen, H. El Gamal, and G. Caire, "On maximum-likelihood detection and the search for the closest lattice point," IEEE Transactions on Information Theory, vol. 49, no. 10, pp. 2389-2402, Oct 2003.

[4] B. Hassibi and H. Vikalo, "On the sphere-decoding algorithm i. expected complexity," IEEE Transactions on Signal Processing, vol. 53, no. 8, pp. 2806-2818, Aug 2005.

[5] L. Lu, G. Li, A. Swindlehurst, A. Ashikhmin, and R. Zhang, "An overview of massive mimo: Benefits and challenges," IEEE Journal of Selected Topics in Signal Processing, vol. 8, no. 5, pp. 742-758, Oct 2014.

[6] C. Ekanadham, D. Tranchina, and E. Simoncelli, "Sparse decomposition of transformation-invariant signals with continuous basis pursuit," in 2011 IEEE International Conference on Acoustics, Speech and Signal Processing (ICASSP), May 2011, pp. 4060-4063.

[7] A. Aissa-El-Bey, D. Pastor, S. Sbai, and Y. Fadlallah, "Sparsity-based recovery of finite alphabet solutions to underdetermined linear systems," IEEE Transactions on Information Theory, vol. 61, no. 4, pp. 2008 2018, April 2015.

[8] Y. Fadlallah, A. Aissa-El-Bey, K. Amis, D. Pastor, and R. Pyndiah, "New decoding strategy for underdetermined mimo transmission using sparse decomposition," in 2013 Proceedings of the 21st European Signal Processing Conference (EUSIPCO), Sept 2013, pp. 1-5.

[9] X. Fan, J. Song, D. P. Palomar, and O. C. Au, "Universal binary semidefinite relaxation for $\mathrm{ml}$ signal detection," IEEE Transactions on Communications, vol. 61, no. 11, pp. 4565-4576, November 2013.

[10] M. Grant and S. Boyd, "CVX: Matlab software for disciplined convex programming, version 2.1," http://cvxr.com/cvx, Mar. 2014.

[11] J. F. Sturm, "Using sedumi 1.02, a matlab toolbox for optimization over symmetric cones," 1998.

[12] I. Gurobi Optimization, "Gurobi optimizer reference manual," 2015. [Online]. Available: http://www.gurobi.com

[13] E. D. Andersen, C. Roos, and T. Terlaky, "On implementing a primaldual interior-point method for conic quadratic optimization," MATHEMATICAL PROGRAMMING SER. B, vol. 95, no. 95, pp. 249-277, 2000.

[14] T. Cui and C. Tellambura, "An efficient generalized sphere decoder for rank-deficient mimo systems," IEEE Communications Letters, vol. 9, no. 5, pp. 423-425, May 2005. 